\title{
Lectura dialógica de álbumes ilustrados para fomentar el pensamiento crítico: un enfoque para Educación Primaria
}

\author{
Dialogic reading of picturebooks to foster critical thinking: an \\ approach for Elementary Education \\ Lectura dialògica d'àlbums il·lustrats per a fomentar el pensament \\ crític: un enfocament per a Educació Primària
}

\author{
Leonor Ruiz-Guerrero. Universidad de Murcia, Spain. Imruiz@um.es \\ https://orcid.org/0000-0003-1520-8368 \\ Beatriz Hoster-Cabo. CEU Cardenal Spínola, Universidad de Sevilla. \\ bhoster@ceuandalucia.es \\ https://orcid.org/0000-0002-7792-7870
}

Sebastián Molina Puche. Universidad de Murcia. smolina@um.es.
https://orcid.org/0000-0003-1469-2100

\begin{abstract}
The discussion of literary texts, and in particular picturebooks, is a valid resource for promoting critical thinking in education (McDaniel, 2006; Roche, 2015; among others). Based on this premise, this paper proposes an approach for dialogic reading, supported by maieutics, which causes a critical look not only at literary aspects but also at the democratic or anti-democratic behaviours present in the picturebooks. The approach integrates, with a writing task, the three critical skills pointed out by Cassany (2017). The methodology is qualitative; it is evaluative research comprising three phases. This article presents the results of the third one, in which the approach was applied to 157 students of the six grades of Elementary Education. The analysis of the responses allows us to contemplate two dimensions: how the students react to the situations that the picturebooks narrate; and how their interventions are produced in the discussion. It is possible to conclude that the guided conversation reinforces the skills of identification and perception of democratic or non-democratic situations, and increases critical abilities such as argumentation, contrasting of ideas, and questioning of what has been read, which become consolidated with writing.
\end{abstract}

Key words: Critical thinking, dialogic reading, Elementary Education, picturebooks. 


\section{Resumen}

La discusión sobre textos literarios, y en particular álbumes, resulta ser un recurso válido para la promoción del pensamiento crítico en el ámbito educativo (McDaniel, 2006; Roche, 2015; entre otros). Desde esa premisa, en este trabajo se propone un enfoque para la lectura dialógica, apoyado en la mayéutica, que provoque una mirada crítica no solo sobre los aspectos literarios, sino también sobre los comportamientos democráticos o antidemocráticos presentes en los álbumes. El enfoque integra, con una tarea de escritura, las tres habilidades críticas señaladas por Cassany (2017). La metodología es cualitativa, se trata de una investigación evaluativa en tres fases. En este artículo se exponen los resultados de la tercera, en la que el enfoque se aplicó a 157 estudiantes de los seis cursos de Educación Primaria. El análisis de las respuestas permite contemplar dos dimensiones: cómo el alumnado reacciona ante las situaciones que los álbumes narran; y cómo se producen sus intervenciones en la discusión. Es posible concluir que la conversación guiada refuerza las habilidades de identificación y percepción de situaciones democráticas o no democráticas, e incrementa las capacidades críticas como la argumentación, contraste de ideas y cuestionamiento de lo leído, que se fijan con la escritura.

Palabras clave: álbumes ilustrados, Educación Primaria, lectura dialógica, pensamiento crítico.

\section{Resum}

La discussió sobre textos literaris, i en particular àlbums, resulta ser un recurs vàlid per a la promoció del pensament crític en l'àmbit educatiu (McDaniel, 2006; Roche, 2015; entre d'altres). Des d'aquesta premissa, en aquest treball es proposa un enfocament per a la lectura dialògica, recolzat en la maièutica, que provoqui una mirada crítica no només sobre els aspectes literaris, sinó també sobre els comportaments democràtics o antidemocràtics presents als àlbums. L'enfocament integra, amb una tasca d'escriptura, les tres habilitats crítiques assenyalades per Cassany (2017). La metodologia és qualitativa, es tracta d'una recerca avaluadora en tres fases. En aquest article s'exposen els resultats de la tercera, en la qual l'enfocament es va aplicar a 157 estudiants dels sis cursos d'Educació Primària. L'anàlisi de les respostes permet contemplar dues dimensions: com l'alumnat reacciona davant les situacions que els àlbums narren i com es produeixen les seves intervencions en la discussió. És possible concloure que la conversa guiada reforça les habilitats d'identificació i percepció de situacions democràtiques o no democràtiques, i incrementa les capacitats crítiques com l'argumentació, contrast d'idees i qüestionament del que s'ha llegit, que es fixen amb l'escriptura.

Paraules clau: àlbums il·lustrats, Educació Primària, lectura dialògica, pensament crític.

\section{Extended abstract}

In these times of post-truth, critical thinking is more necessary than ever. If we want to strengthen our democracies and save them from the threats looming over them (Alba Rico et al., 2017; Mounk, 2018; Todorov, 2016; Valadier, 2017), this kind of thinking must be fostered as an unavoidable priority.

Schools are the most appropriate place to promote critical thinking, since democracy is a content that can be learned in the educational context both through reflection and practice. However, for a long time, it was considered that critical thinking, being a higher-order skill, could not be acquired at lower educational levels, such as Elementary Education. Fortunately, new winds of change are blowing that denote the contrary (Álvarez-Álvarez y Pascual-Díez, 2013; Chambers, 2017; Evans, 2015; Guzmán-Cedillo y Flores, 2020; Jurado, 2008; Klooster, 2001; Roche, 2015), and even the educational legislation integrates critical thinking as a core objective in the cited stage.

There are several strategies to promote this type of thinking in education. In this paper, we want to explore the discussion of literary texts, and in particular picturebooks, as a valid resource (McDaniel, 2006; Roche, 2015; among others). Based on this premise, this paper proposes an approach for dialogic reading, supported by maieutics, which generates a critical look not only at literary aspects but also at the 
democratic or anti-democratic behaviours present in the picturebooks. This work studies the connection between visual/verbal mechanisms and the generation of critical thinking in this kind of text, adding to the already existing theoretical corpus (Chambers, 2015; Cunningham y Enriquez, 2013; Da Mota, 2020; Evans, 2015; Kalogirou y Malafantis, 2014; McDaniel, 2004, 2006; Peterson y Eeds, 2007; Roche, 2015). Through a writing task, the approach integrates the three critical skills pointed out by Cassany (2017): thinking, reading, and writing. Moreover, it allows to address the traits that Klooster (2001) incorporates into this kind of thinking: its independent or individual character, information as to its starting point and not its point of arrival, its initiation with questions or problems to be solved; its pursue of reasoned arguments, and its social nature.

The methodology of the study is qualitative, being an evaluative research comprising three phases. This article presents the results of the third one, in which the approach was applied to 157 students of the six grades of Elementary Education from one Spanish public school. The didactic intervention was structured in three sessions. First, the students were familiarized with the approach using an introductory picturebook; then, they collectively explored the selected picturebook, and finally, they individually wrote a text adopting the stance of the characters. The titles elected were Bugs in a Blanket (Alemagna, 2011), for the first grade; Four Little Corners (Ruillier, 2005), for the second grade; The Conquerors (McKee, 2004), for the third grade; The Hueys in the New Jumper (Jeffers, 2015), for the fourth grade; Louis I, King of the sheep (Tallec, 2015), for the fifth grade; and The Enemy (Cali, 2008), for the sixth grade.

The approach presented is based on Tell me, by Chambers (2017) and Critical Thinking and Book Talk (CT\&BT), by Roche (2015). Furthermore, it considers the five functions of mediation that teachers assume in this type of discussion, detailed by Munita and Manresa (2012): to help in the search for significant textual clues; to help in the construction and substantiation of their arguments; to relate the discussion to other books and previous knowledge; to offer metalanguage to talk about books; and to reformulate, synthesize and systematize what has been said to advance the discussion and to fix concepts and contents. In the proposed approach, the first two functions have had significant relevance. Last but not least, it also relies on the Picturebook triangle model by Ellis and Mourão (2021).

The approach suggested has four steps: an introduction (before reading), a start-up phase, a development and a closing phase (after reading). In the development phase, the mediator offers the group several questions that follow the typology collected by Kalogirou and Malafantis (2012) for this kind of discussion. From this phase onwards, aspects are introduced that represent a novelty concerning previous studies. The first of these would be the concreteness of the questions, which, without contradicting their open nature, allows students to identify what they should focus their attention on. The first question requires them to place themselves in the conflictive situation that the picturebook renders, recognizing it. After this, the questions are structured around three dimensions: difference/diversity, conflict resolution, and the visual/textual ellipses of the picturebook. The formulation of the questions aims at making inferences, expressing argued opinions, and hypothesizing, both about what is narrated and about the author's intentionality. The second innovative aspect is presented in the closing phase, in which students are expected to transcend the text.

The analysis of the responses allows us to contemplate two dimensions: how the students react to the situations that the picture books narrate; and how their interventions are produced in the discussion. It is possible to conclude that the guided conversation reinforces the skills of identification and perception of democratic or non-democratic situations, and increases critical abilities such as argumentation, contrasting of ideas, and questioning of what has been read, which become consolidated with writing. 


\section{Introducción}

El desarrollo del pensamiento crítico, junto con el de otros elementos de ciudadanía democrática, es una preocupación que se extiende por Europa desde, al menos, finales del siglo XX. Acciones como el auge de movimientos populistas y radicales de diverso signo político o la manipulación de la información, son difíciles de contener sin la presencia de este pensamiento y llevan a un temor creciente a que se produzca el desmantelamiento de los sistemas democráticos (Alba Rico et al., 2017; Mounk, 2018; Todorov, 2016; Valadier, 2017).

Frente a este temor, el ámbito educativo se erige como el espacio idóneo para recuperar el sentido de la democracia. Es preciso que desde las aulas, y desde bien temprano, se enseñe a ser ciudadanía (Santisteban, 2004). Sin embargo, durante mucho tiempo ha existido la creencia de que por ser una habilidad de orden superior el pensamiento crítico no podía ser adquirido en los niveles educativos inferiores, como Educación Primaria. La persistencia de esta idea se observa en el último informe Eurydice, donde se

[...]durante mucho tiempo ha existido la creencia de que por ser una habilidad de orden superior el pensamiento crítico no podía ser adquirido en los niveles educativos inferiores, como Educación Primaria. constata que en la organización de la educación cívica en Europa este pensamiento se desplaza a la Educación Secundaria (European Commission, 2017). Asimismo, el corpus teórico se inclina hacia esta etapa y la universitaria (Albertos y De la Herrán, 2018; Martínez, Ballester e Ibarra, 2018). Empero, se perciben también cambios que se reflejan en la legislación. En el contexto español, el Real Decreto 126/2014 para Educación Primaria nombra repetidamente el sentido crítico, se señala como un objetivo de la etapa, y se incluye en los elementos curriculares de las áreas de Ciencias Sociales y Lengua Castellana y Literatura. Hay también un interés al alza desde el campo investigador (ÁlvarezÁlvarez y Pascual-Díez, 2013; Chambers, 2017; Evans, 2015; Guzmán-Cedillo y Flores, 2020; Jurado, 2008; Roche, 2015). Como Klooster (2001, p. 38) asevera: "students of every age, from the first grade to the university, can think critically, because all of them already have rich life experiences and deep resources of prior knowledge."

Ante la variedad de perspectivas existentes sobre qué es pensar críticamente, este artículo se decanta por los cinco rasgos propuestos por el citado Klooster (2001). Para él este pensamiento posee un carácter independiente o individual; la información es su punto de partida, no de llegada; se inicia con preguntas, problemas por resolver; persigue argumentos razonados; y es social. Estos atributos son subrayados también, total o parcialmente, por otros autores (Cassany, 2017; Jurado, 2008), y beben en gran medida de la pedagogía crítica de Freire (Morales, 2018) y Giroux (1990). La 
relevancia del aspecto social es remarcada también por Mercer (1997), que aboga por fomentar conversaciones de tipo exploratorio en las aulas, ya que estas generarán un pensamiento compartido (interthinking) fruto de la intersección crítica y argumentada de las voces participantes.

Para alcanzar el pensamiento crítico son diversos los recursos y estrategias que se pueden emplear; en este estudio se propone el uso de textos literarios. Enseñar a leerlos con mirada alerta es el fin último de esta investigación. Como afirma Cassany (2017, p. 114): "Ante el mundo multicultural, globalizado, dinámico y conflictivo en el que vivimos, la única respuesta educativa posible es la necesidad de formar a una ciudadanía autónoma y democrática que tenga habilidades críticas de lectura, escritura y pensamiento."

El vínculo entre literatura y pensamiento crítico, y su abordaje en las aulas, ha sido planteado de manera abundante (Álvarez-Álvarez y Pascual-Díez, 2013; Cassany, 2004, 2017; Freebody y Luke, 1991; Jurado, 2008; Saldaña, 2020). La singularidad de los textos literarios parece hacerlos especialmente favorables al logro de tal pensamiento (Jurado, 2008). Esto ocurre de manera significativa en el género álbum ilustrado, debido a su mayor apertura interpretativa, que posibilita la puesta en común y contraste de diferentes perspectivas. El presente trabajo considera la conexión en esta clase de obras entre los mecanismos visuales/verbales y la generación de pensamiento crítico, sumándose al corpus teórico ya existente (Chambers, 2015; Cunningham y Enriquez, 2013; Da Mota, 2020; Evans, 2015; Kalogirou y Malafantis, 2014; McDaniel, 2004, 2006; Peterson y Eeds, 2007; Roche, 2015). En estos estudios se pone el acento tanto en la lectura como en la discusión literaria posterior.

La lectura dialógica, o discusión literaria, se conforma en una estrategia de primer orden para alcanzar los propósitos aquí señalados. Se trata de animar al alumnado a conversar sobre lo leído, aportando sus puntos de vista de manera argumentada. Quien modera la sesión sugiere una serie de interrogantes como germen de la discusión. Estas preguntas suelen ser en un primer momento genéricas: ¿qué te ha gustado de lo leído?, ¿alguien tiene algo interesante que decir?; y más tarde específicas. Se trata de una labor de mediación basada en estrategias de andamiaje (scaffolding). Ellis y Mourão (2021) lo muestran a través del Picturebook triangle model, que tiene la siguiente estructura: 


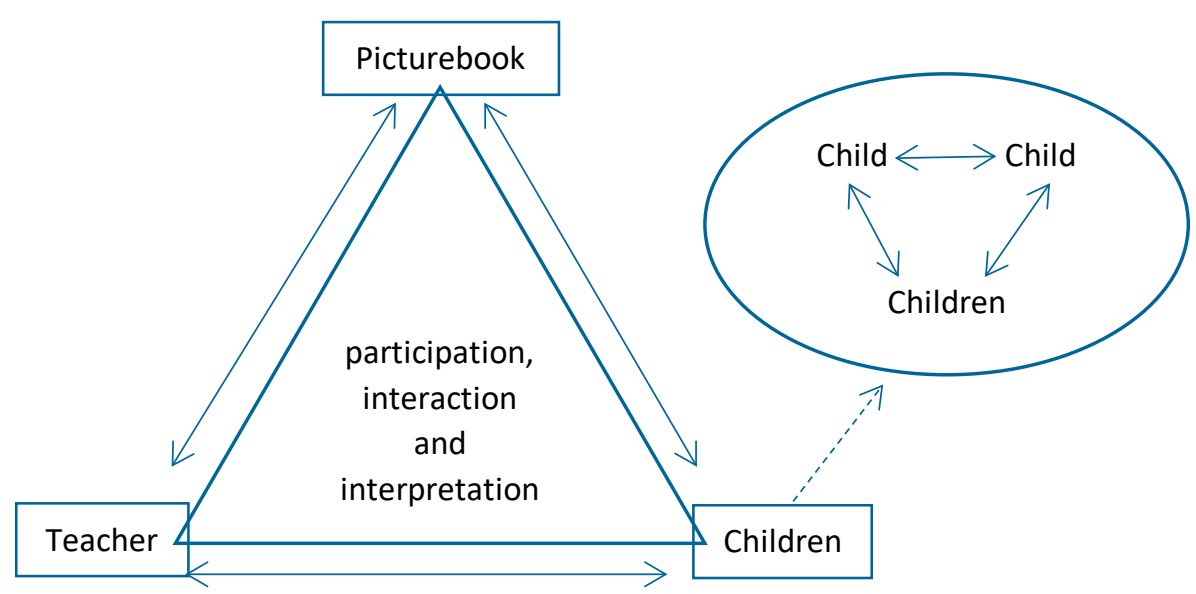

Figura 1. Estructura de The picturebook triangle model. Fuente: Ellis y Mourão (2021).

Cuando esta discusión tiene como fin el despliegue del pensamiento crítico, lo que se promueve es una posición de cuestionamiento sobre el texto, que pueda trasladarse al resto de ámbitos de la vida. Basándose en Burke (2010), Kalogirou y Malafantis (2012) recogen la tipología más idónea de interrogantes para este tipo de discusión: "1. Factual or verifiable questions that respond to matters of who, when, what, where, how?" (p. 268); "2. Inductive

El estudio que aquí se muestra sigue la estela de los previos, pero pretende innovar con la oferta de un enfoque para la discusión cuyos interrogantes, si bien parten de lo literario, abren la puerta a reflexiones de tipo cívico, a pensar críticamente sobre la vida social y sus bases democráticas questions that are still verifiable but respond to matters of why, how, so what?" (p. 269); y "3. Analytical questions that connect the text to other texts, ideas or situations." (p. 269) ${ }^{1}$. De ellos se deduce que la lectura y discusión crítica sobre un texto no se ciñe solo a este, sino que demanda la contextualización de la obra en unos parámetros sociohistóricos que condicionan su interpretación (Cassany, 2013).

Desde el rol moderador también se proponen pautas para un intercambio comunicativo basado en los principios democráticos, tales como el respeto a las ideas ajenas, o el uso del turno de palabra (Roche, 2015). De este modo, se ayuda a comprender que en democracia la diversidad de opiniones no solo es aceptable, sino deseable, pero que debe ser canalizada a través del diálogo respetuoso.

Los resultados de las investigaciones sobre esta lectura dialógica evidencian su efectividad, e incitan a continuar con su empleo, pero también a buscar aplicaciones que traspasen la frontera de la crítica o conocimiento puramente literarios. El estudio que aquí se muestra sigue la estela de los previos,

\footnotetext{
${ }^{1}$ La cursiva es del texto original.
} 
pero pretende innovar con la oferta de un enfoque para la discusión cuyos interrogantes, si bien parten de lo literario, abren la puerta a reflexiones de tipo cívico, a pensar críticamente sobre la vida social y sus bases democráticas.

\section{Metodología}

El presente estudio se inserta en el paradigma cualitativo, como sus precedentes investigadores. El diseño del enfoque se encuadra dentro de una investigación de mayor envergadura desarrollada en tres fases de trabajo de campo. De carácter evaluativo, en ella se ha empleado el modelo CIPP (Stufflebeam y Shinkfield, 1995), compuesto por cuatro evaluaciones: contexto, entrada, proceso y producto. Se escogió este tipo de investigación por ser adecuada a los fines perseguidos: comprobar la validez de un programa aplicado en centros educativos. Las diversas evaluaciones permitieron el perfeccionamiento de dicho programa, llegando a la versión definitiva concretada en el enfoque que se va a exponer en este artículo.

La pregunta de investigación formulada en este estudio fue: ¿cómo podría el alumnado de Educación Primaria extraer aprendizajes de los álbumes referidos a contenidos de educación cívica? De ella se deriva el siguiente objetivo: comprobar la eficacia del empleo de los álbumes para el avance en la adquisición de contenidos de Ciencias Sociales relacionados con las competencias sociales y cívicas, representados en un soporte ficcional, así como de formas literarias propias de este soporte.

\subsection{Contexto y fases de la investigación}

Dos centros de Educación Primaria, de una sola línea y titularidad pública, ubicados en la Región de Murcia (Centro 1) y Andalucía (Centro 2) participaron de la aplicación del programa, que se desarrolló en tres fases (Tabla 1). Un total de 587 estudiantes de Educación Primaria, de todos los cursos, se implicaron en la lectura de los álbumes.

\begin{tabular}{|c|c|c|c|}
\hline Fase & Curso & Centro & Sesiones (45-50 minutos) \\
\hline Primera & $2015 / 16$ & Centro 1 (experiencia piloto) & 6 \\
\hline Segunda & $2017 / 18$ & Centros 1 y 2 & 6 \\
\hline Tercera & $2018 / 19$ & Centro 1 & 3 \\
\hline
\end{tabular}

Tabla 1. Fases y contexto de la investigación

Las dos primeras fases sirvieron para comprobar la idoneidad de las primeras actividades planteadas, apoyadas en las ofrecidas en el corpus académico existente, que solían incluir una lectura en voz alta por parte de quien dirige la sesión, seguida de una serie de tareas orales, escritas 
o gráficas con las cuales se da respuesta a lo leído/escuchado (Arizpe y Styles, 2004; Colomer, 2012; Courtland y González, 2013; Evans, 2015; Phipps, 2016).

En la tercera fase, que es de la que se hablará en este artículo, tomaron parte 157 estudiantes (Tabla 2).

\begin{tabular}{|l|c|c|c|}
\hline \multicolumn{1}{|c|}{ Curso } & Niños & Niñas & Total \\
\hline Primero & 14 & 12 & 26 \\
\hline Segundo & 12 & 14 & 26 \\
\hline Tercero & 12 & 15 & 27 \\
\hline Cuarto & 17 & 10 & 27 \\
\hline Quinto & 13 & 13 & 26 \\
\hline Sexto & 15 & 10 & 25 \\
\hline
\end{tabular}

Tabla 2. Alumnado participante en la 3 a fase del trabajo de campo (Centro 1 )

En un primer momento los contenidos de educación cívica abordados eran los relativos a la aceptación y respeto de la diversidad, la resolución pacífica de conflictos, y el pensamiento crítico. Pronto se observó que sin el último no pueden darse los primeros, por lo que se constituyó en nuclear e integrador de los otros dos.

En las dos primeras fases del trabajo de campo se obtuvieron respuestas por parte del alumnado ajustadas a lo deseable, en los diferentes instrumentos de recogida de datos (cuestionarios sobre paratextos, y sobre elementos del texto; ficha de análisis sobre mecanismos empleados en el álbum para estimular el pensamiento crítico; y producciones gráficas y escritas poniéndose en el lugar de los personajes). Asimismo, tanto el grupo de discusión con el profesorado del Centro 1, como el método Delphi empleado con el del Centro 2, arrojaron una valoración positiva del programa. Sin embargo, se apreció que los resultados eran susceptibles de mejora, ya que las actividades propuestas parecían no dejar suficiente espacio a la argumentación y producían redundancia en las contestaciones.

Se decidió entonces considerar los parámetros de la lectura dialógica. El valor de las discusiones, como revela Rincón (2012), no reside solo en el discurso resultante, sino también en el proceso mismo de llegar a él. Las estrategias de análisis empleadas, los andamiajes que el propio alumnado se proporciona entre sí, los conocimientos previos poseídos, emergen en la conversación.

\subsection{Diseño del enfoque}

Con este enfoque se pretendía reorientar la intervención de las primeras fases, reduciendo el aura de trabajo escolar y facilitando al profesorado un modo de actuación claro pero flexible. Al adoptar la conversación mayor protagonismo, los docentes se convierten en mediadores de lectura. Munita y Manresa (2012) detallan las cinco funciones de la mediación que el profesorado asume en la 
discusión de este tipo: ayudar en la búsqueda de indicios textuales significativos; ayudar en la construcción y fundamentación de sus argumentaciones; relacionar la discusión con otros libros y saberes previos; ofrecer metalenguaje para hablar sobre libros; y reformular, sintetizar y sistematizar lo dicho para hacer progresar la discusión y para fijar conceptos y contenidos. En el enfoque propuesto, van a tener especial relevancia las dos primeras funciones. La segunda, además, tiene relación directa con el desarrollo del pensamiento crítico. Ya que:

El mediador ayuda al lector no sólo a interpretar el sentido de las historias sino a entender la manera en la que están contadas, pues "obliga" a indagar y a reflexionar sobre la función de ciertos recursos literarios o visuales en la narración. (Munita y Manresa, 2012, p. 127)

Aparte de los citados Munita y Manresa (2012), para el diseño se han tomado como referentes el enfoque Dime (Chambers, 2017), y el método CT\&BT (Critical Thinking and Book Talk), de Mary Roche (2015). Chambers (2017) confía en el potencial de análisis crítico del alumnado, que se manifiesta cuando pone sus pensamientos en interrelación. Roche (2015), por su parte, hace más hincapié en la propia interacción comunicativa: turno de palabra y expresión del acuerdo o desacuerdo con el resto. Ambos autores exponen una serie de preguntas para iniciar la conversación.

El enfoque se concreta a partir de estos modelos de discusión y de la síntesis de los instrumentos para recogida de datos utilizados en las etapas previas de

Con la inclusión de tal actividad, el enfoque recoge las tres habilidades críticas señaladas por Cassany (2017): lectura, escritura y pensamiento. Este nuevo diseño del programa posibilita, además, recuperar las cinco cualidades definitorias del pensamiento crítico expuestas por Klooster (2001). aplicación del programa: cuestionarios sobre paratextos (cubierta y contracubierta) y elementos del álbum (personajes, acciones, etc.); así como ficha de análisis sobre los mecanismos empleados en las obras para la promoción del pensamiento crítico. El resultado es un conjunto de preguntas abiertas que son el núcleo de la conversación. Este modelo de intervención se compone de una introducción y tres fases, que pueden tener lugar en una única sesión, o ser distribuidas en varias. De hecho, Chambers (2017) propone un espacio de un fin de semana entre la lectura y la conversación sobre un texto.

La lectura dialógica con el enfoque diseñado se desarrolla en gran grupo, y tras la misma se sugiere una actividad de redacción individual, que permita consolidar los aprendizajes. Planteada ya en las fases previas del trabajo de campo, en ella el alumnado se posiciona en el lugar de los personajes del 
álbum leído. Según el curso esta tarea se apoya más en el dibujo o en la escritura, aumentando la complejidad conforme se avanza en la etapa educativa. Con la inclusión de tal actividad, el enfoque recoge las tres habilidades críticas señaladas por Cassany (2017): lectura, escritura y pensamiento. Este nuevo diseño del programa posibilita, además, recuperar las cinco cualidades definitorias del pensamiento crítico expuestas por Klooster (2001).

A continuación, se describe pormenorizadamente el diseño definitivo del enfoque, indicando qué ha de hacer la persona que dirige la sesión:

Introducción (previo a la lectura)

- Se muestra la cubierta, contracubierta del álbum y guardas, y se pregunta qué se ve en ellas. Se escuchan las respuestas. No se hacen correcciones (se puede orientar con nuevas preguntas).

- Se pregunta de qué puede tratar la historia y qué les hace creer eso. Se escuchan las respuestas sin realizar correcciones.

- Quien dirige la sesión lee el álbum en voz alta. Durante la lectura, se permite la participación del alumnado (expresión de ideas, opiniones, expectativas), además se pueden proponer preguntas sobre lo que se dice en el texto y lo que se muestra en las ilustraciones.

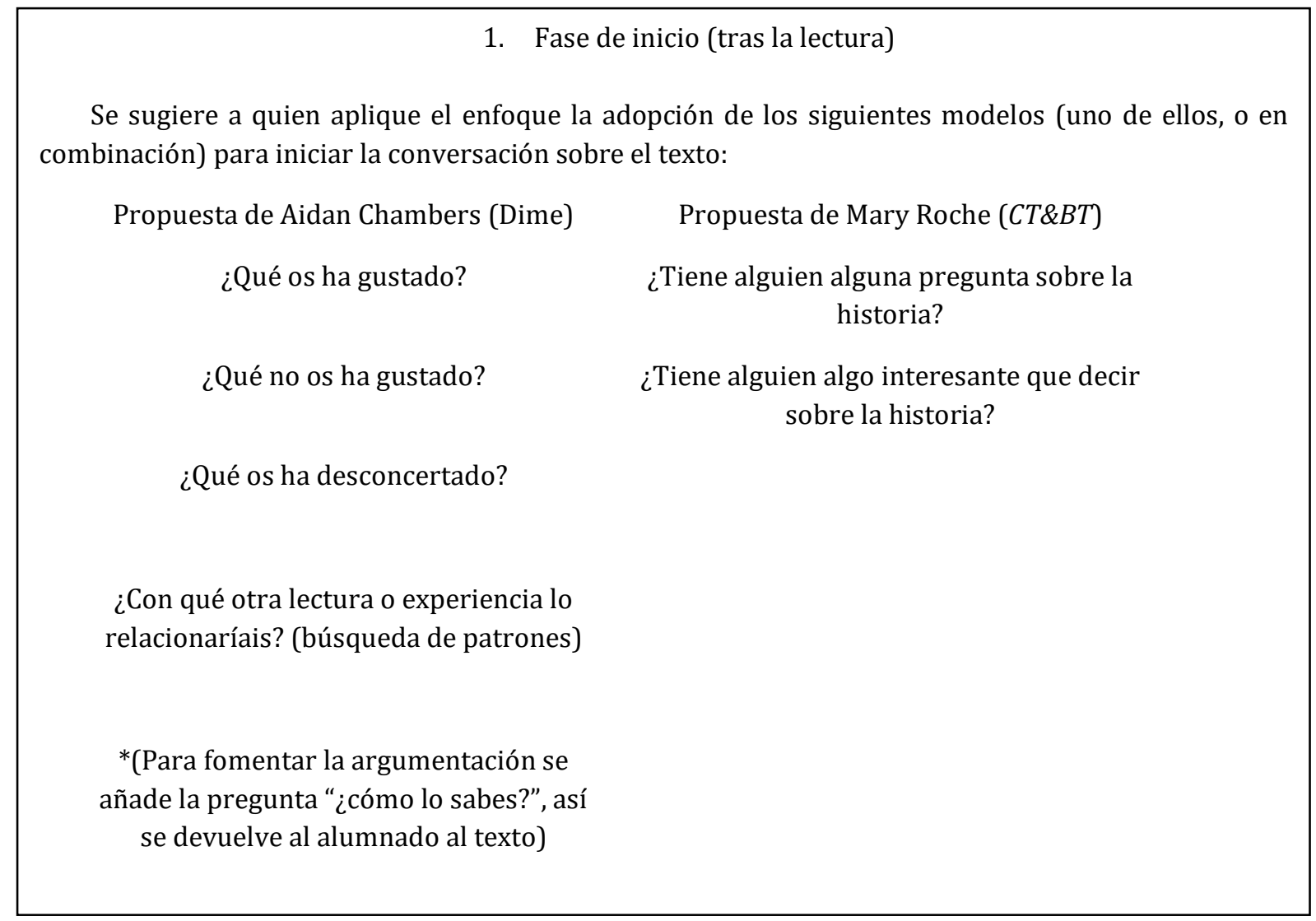


En esta introducción y primera fase se podría decir que el diálogo se produce sobre todo entre el texto y cada participante de la discusión. No obstante, quien dirige la sesión puede procurar el avance hacia el diálogo entre discentes, recurriendo a la quinta función de mediación de Munita y Manresa (2012).

\section{Fase de desarrollo (tras la lectura)}

Quien dirige la sesión continúa con las siguientes cuestiones:

Parece que en este libro los personajes tienen un problema, ¿cuál es?

¿Cómo son los personajes? ¿Se parecen entre sí? ¿Cómo lo sabéis? ¿A los personajes les gusta, no les gusta, o les da igual lo de ser diferentes (o lo de ser iguales)?

¿Hay algún personaje que se crea diferente por algo que ha pasado en el libro? ¿0 que los demás digan que es diferente (puede decirlo el propio álbum)? ¿Creéis que es de verdad diferente? ¿Ha pasado algo en la historia que haya hecho que ya no sea diferente y se vuelva como los demás? ¿0 que ahora piense de otra manera sobre ser diferentes?

¿Cómo han resuelto el problema que tenían? ¿Qué ha hecho este/os personaje/s? ¿Y los otros? ¿Han hablado en algún momento para resolverlo? ¿Os gusta cómo lo han resuelto? ¿Todos los personajes están de acuerdo con la solución?

¿Hay algo que no nos hayan contado en el libro, sobre lo que pasa en él? ¿Esta historia termina, hay un final? ¿Por qué lo habrá hecho así quien ha escrito el libro? ¿Esperabais que pasase eso en la historia? ¿Qué creíais que iba a pasar?**

**Estas preguntas son orientativas, cada docente ha de adecuarlas a su alumnado. No necesariamente hay que hacerlas todas, ni en ese orden. Dependerá tanto del alumnado como del texto que se ha manejado. Por otra parte, estas preguntas se formulan solo oralmente, y no se proporcionan al grupo.

\section{Fase de cierre (tras la lectura)}

Quien dirige la sesión propone una cuestión que sirva para extraer conclusiones:

¿Qué podemos aprender con este libro?

Las fases dos y tres suponen la expansión del diálogo grupal, la plenitud del modelo triangular de Ellis y Mourão (2021). Por otra parte, a partir de la Fase de desarrollo se introducen aspectos novedosos respecto a los estudios previos. El primero de ellos sería la concreción de las preguntas, que sin contrariar su carácter abierto, permite al alumnado identificar con claridad aquello en lo que debe focalizar su atención. La primera pregunta requiere que se ubique en la situación conflictiva que el álbum propone, reconociéndola. Tras esto los interrogantes se vertebran alrededor de tres dimensiones: la diferencia/diversidad; la resolución de conflictos; y las elipsis visuales/textuales del 
álbum. La formulación de las cuestiones pretende la realización de inferencias, la expresión de opiniones argumentadas, y el planteamiento de hipótesis, tanto sobre lo narrado como sobre la intencionalidad del autor/a. El segundo aspecto innovador se presenta en la Fase de cierre, en la que se espera que el alumnado transcienda el texto.

Los interrogantes de estas dos últimas fases se ajustan a la tipología expuesta por Kalogirou y Malafantis (2012) a partir de Burke (2010). Por ejemplo, las preguntas relativas a cuál es el problema y a cómo son los personajes serían de índole fáctica o verificable, mientras que cuestiones tales como si todos los personajes están de acuerdo con la solución dada podrían etiquetarse como inductivas. Asimismo, otras tendrían carácter analítico, como la de qué creíais que iba a pasar [en la historia].

\subsection{Procedimiento}

El desarrollo del enfoque en la tercera fase del trabajo de campo se organizó del siguiente modo (Tabla 3):

\begin{tabular}{|c|c|c|c|c|}
\hline & Objetivo & Actividad & $\begin{array}{l}\text { Instrumento de } \\
\text { recogida de datos }\end{array}$ & Agrupamiento \\
\hline 1a sesión & $\begin{array}{l}\text { Familiarizar con } \\
\text { el enfoque }\end{array}$ & $\begin{array}{l}\text { Lectura y conversación } \\
\text { sobre álbum de } \\
\text { temática similar al } \\
\text { seleccionado }\end{array}$ & Notas de campo & Gran grupo \\
\hline 2ª sesión & $\begin{array}{l}\text { Aplicar el } \\
\text { enfoque al álbum } \\
\text { seleccionado }\end{array}$ & $\begin{array}{l}\text { Lectura y conversación } \\
\text { sobre álbum } \\
\text { seleccionado }\end{array}$ & $\begin{array}{l}\text { Grabación de la } \\
\text { discusión y } \\
\text { transcripción }\end{array}$ & Gran grupo \\
\hline 3a sesión & $\begin{array}{c}\text { Fijar } \\
\text { aprendizajes }\end{array}$ & $\begin{array}{l}\text { Redacción de texto } \\
\text { poniéndose en el lugar } \\
\text { de los personajes }\end{array}$ & $\begin{array}{l}\text { Producciones del } \\
\text { alumnado }\end{array}$ & Individual \\
\hline
\end{tabular}

Tabla 3. Organización de las sesiones en la tercera fase del trabajo de campo

Todos los datos recogidos se analizaron con el programa Atlas.ti. La selección de los álbumes para cada curso de la etapa siguió criterios de actualidad, accesibilidad, calidad, adecuación a los participantes e idoneidad para los fines perseguidos. Los títulos son El país de las pulgas (Alemagna, 2011), para primer curso; Por cuatro esquinitas de nada (Ruillier, 2005), para segundo; Los conquistadores (McKee, 2004), para tercero; Los Huguis en El jersey nuevo (Jeffers, 2015), para cuarto; Felicio Rey del rebaño (Tallec, 2015), para quinto; y El enemigo (Cali, 2008), para sexto.

\section{Análisis y resultados}

La identificación de las manifestaciones de pensamiento crítico en las sesiones de lectura dialógica se ha articulado en torno a dos dimensiones, ambas con una serie de códigos. Este proceso de 
categorización se ha realizado a partir del análisis de la transcripción de las grabaciones en audio, obteniendo 30 códigos en total.

La primera dimensión está vinculada al propio texto y a cómo el alumnado reacciona ante la diversidad, los conflictos y la ausencia de sentido crítico de los personajes. En la Tabla 4 se presentan los códigos más relevantes de esta dimensión.

Categoría

Resolución de conflictos
Pensamiento crítico

\section{D1.1. Percepción de la diferencia: reconoce que hay personajes diferentes en el texto, y cómo se exhibe tal diferencia.}

\begin{abstract}
Aceptación de la diversidad

D1.2. Identificación de la reacción ante la diversidad: percibe si hay rechazo o aceptación de la diversidad en el álbum, incluyendo si hay una evolución de una actitud a la otra.
\end{abstract}

D1.3. Opinión favorable a la diversidad: se muestra a favor de la diferencia, rechazando la uniformidad.

D1.4. Reconocimiento del conflicto: se percata de la presencia de un conflicto entre los personajes, que debe ser resuelto.

D1.5. Rechazo de la violencia: se posiciona en contra de las resoluciones violentas, por sus consecuencias para los personajes.

D1.6. Valor del diálogo para resolver el conflicto: resalta el diálogo como la forma más adecuada de resolución.

D1.7. Toma de conciencia del engaño: percibe cómo unos personajes engañan a otros para conseguir un beneficio particular.

D1.8. Rechazo a las imposiciones: se muestra disconforme con las imposiciones de todo tipo de unos personajes sobre otros.

D1.9. Importancia de tener criterio: se da cuenta de que sin criterio los personajes son fácilmente manipulables. Contrapone la capacidad para tomar decisiones por uno mismo.

Tabla 4. Códigos para la $1 \underline{\text { a }}$ dimensión. Reacciones del alumnado ante lo narrado

La segunda dimensión está ligada al modo en que se producían las intervenciones en la discusión. De entre todos los códigos resultantes destacan (Tabla 5):

\section{Códigos}

\section{D2.1. Divergencia respecto a lo opinado por otras personas: el alumnado expresa su} desacuerdo, de forma argumentada, con respecto a otras opiniones.

\section{D2.2. Explicación extensa: el alumnado argumenta sus ideas, yendo más allá de la respuesta} breve o monosilábica.

D2.3. Intercambio basado en el conocimiento previo: el alumnado, a la hora de plantear sus ideas, se apoya en lo que ya sabe sobre la temática de los textos (por experiencia, por conexiones intertextuales, por vínculos extraliterarios, etc.). 
D2.4. Reajuste de ideas tras contraste: el alumnado modifica y precisa sus opiniones tras ponerlas en contacto con las de otros y descubrir que deben ser reajustadas.

\section{D2.5. Recogida de intervenciones ajenas: el alumnado recupera ideas de otras personas del grupo para ampliarlas, rebatirlas...}

D2.6. Aprendizaje extraíble: el alumnado expresa qué aprendizajes pueden recabarse tras la lectura del álbum.

Tabla 5. Códigos para la 2ª dimensión. Intervenciones durante la discusión

Para ejemplificar estas dimensiones y algunos de sus códigos, se reproducen en primer lugar varios fragmentos de las conversaciones sostenidas en los diferentes cursos (Tabla 6). En estas citas se puede apreciar que los códigos se interrelacionan.

\begin{tabular}{|c|c|}
\hline Códigos & Cita de ejemplo \\
\hline $\begin{array}{l}\text { D1.1. Percepción de la } \\
\text { diferencia } \\
\text { D1.3. Opinión favorable } \\
\text { a la diversidad }\end{array}$ & $\begin{array}{l}\text { Alumna 13: Pues lo que no me gusta es que todos fueran igual } \\
\text { Coordinadora: Que todos fueran iguales, eso no te ha gustado. Pero luego } \\
\text { eran diferentes, ¿no? } \\
\text { Alumna 1: Siguen siendo iguales, porque tienen el mismo jersey } \\
\text { Coordinadora: Ah, pero dicen que son diferentes } \\
\text { Alumna 13: Pero no lo son ( } 4^{\circ} \text { curso) }\end{array}$ \\
\hline $\begin{array}{l}\text { D1.5. Rechazo de la } \\
\text { violencia }\end{array}$ & $\begin{array}{l}\text { Alumna 17: A mí lo que menos me ha gustado ha sido cuando estaba } \\
\text { diciendo uno, vale, pues tú eres un plátano, tú eres... porque eso es } \\
\text { Alumna 26: Insultar } \\
\text { Alumna 17: Insultos (1.er curso) }\end{array}$ \\
\hline $\begin{array}{l}\text { D1.8. Rechazo a las } \\
\text { imposiciones } \\
\text { D2.3. Intercambio } \\
\text { basado en el } \\
\text { conocimiento previo }\end{array}$ & $\begin{array}{l}\text { Coordinadora: ¿Pero ha echado a las amarillas y a las blancas? } \\
\text { Varios: ¡A las negras! } \\
\text { Varios: ¡Racista! [Se refieren al personaje de Felicio] } \\
\text { [...] } \\
\text { Alumno 5: Que la oveja esa es como Donald Trump } \\
\text { Coordinadora: Es igual que Donald Trump. ¿Por qué dices que es como } \\
\text { Donald Trump? Quiero oír a vuestro compañero, y creo que el resto } \\
\text { también querrá oírlo porque estaba diciendo una idea que no ha dicho } \\
\text { antes nadie } \\
\text { Grupo: ¿Qué? } \\
\text { Coordinadora: Cree que se parece a Donald Trump. ¿Creéis que se parece? } \\
\text { Grupo: [risas] } \\
\text { Varios: Es Donald Trump (5ํo curso) }\end{array}$ \\
\hline $\begin{array}{l}\text { D2.2. Explicación } \\
\text { extensa } \\
\text { D2.5. Recogida de } \\
\text { intervenciones ajenas } \\
\text { D2.4. Reajuste tras } \\
\text { contraste }\end{array}$ & $\begin{array}{l}\text { Alumno 18: Placas, y, pues aquí... pues tiene como... sangre en la mano } \\
\text { Alumno 20: No es sangre, es una... } \\
\text { [...] } \\
\text { Coordinadora: A ver, mirad, } 18 \text { ha dicho que tiene sangre en las manos, } \\
20 \text { dice que no. ¿Por qué pensáis que...? } \\
\text { Alumno 20: Porque son como una especie de estas, de... } \\
\text { Coordinadora: No, pero en las manos, fíjate en las manos, las manos } \\
\text { Alumno 20: Ah, las manos. Ah, es que no se... ¡Es verdad, lleva sangre! } \\
\text { Alumno 18: Creo que acaba de matar a alguien } \\
\text { Coordinadora: ¿Es la sangre de otra persona? } \\
\text { Alumno 20: El enemigo ha matado a alguien con mucha sangre en las } \\
\text { manos. Es un enemigo ( } 6 \text { co curso) }\end{array}$ \\
\hline
\end{tabular}

Tabla 6. Ejemplos de manifestaciones de pensamiento crítico 
Respecto a la diferencia/diversidad, en los álbumes suele presentarse en los personajes a partir del color, las formas, la posesión de un atributo, o bien de modo sutil siendo construida en el discurso pero no visible. A veces es contrastada de forma irónica con la igualdad clónica para que sea más evidente, como ocurre en Los Huguis en El jersey nuevo (Jeffers, 2015), leído en 4o curso. La uniformidad suele provocar rechazo en el alumnado, sobre todo si se detecta como imposición o falta de criterio. Así se observa en primer curso, cuando un personaje espera que el resto sea como él:

\author{
Alumna 23: Porque es que las pulgas no tienen que ser iguales que la pulga gorda, porque \\ si no cada, si no van a ser todas iguales y a mí tienen que ser diferentes \\ Alumna 26: Sí, porque todas igualicas no son muy bonitas \\ Coordinadora: ¿No? Es mejor... ¿ ¿te gustan a ti más de colorines? \\ Alumna 26: Sí
}

Aunque no todo el alumnado alcanza el mismo grado de pensamiento crítico, el intercambio y acceso a las ideas de quienes son más capaces de leer entre y detrás de las líneas tiene una fuerza generativa en el resto. En el fragmento que se reproduce a continuación, correspondiente a 6으 curso, se pone de manifiesto. Un primer estudiante plantea una hipótesis sobre lo que está ocurriendo en la narración, otro recuerda lo que se dice en el manual entregado a los soldados protagonistas del álbum ("es una bestia despiadada"), y más tarde realiza una afirmación que emerge de una inferencia ("ni él tampoco"). Mientras, otros dos discentes extraen conclusiones, que se entretejen con lo dicho por sus compañeros y lo leído/visto. Este breve diálogo revela la doble cualidad individual y social del pensamiento crítico (Klooster, 20021):

Alumno 14: Porque los jefes son los malos, le engañan a... Que los jefes son los malos, los jefes son los que han hecho que uno se equivoque, se confundan entre los dos y dispare, con el propósito de divertirse ellos viendo cómo se disparan, engañando y poniendo el mismo manual a cada uno para que crean que el otro es así

Alumno 20: Es una bestia despiadada

Coordinadora: Vamos a ver

Otro alumno: No hay ninguno malo ni ninguno bueno

[Cuando el personaje encuentra el manual, y lo rechaza porque miente.]

Alumno 19: Es el mismo manual 
[El soldado asegura que no ha matado a mujeres ni niños]

Alumno 20: Ni él tampoco [refiriéndose al enemigo]

Por otra parte, estos niños y niñas son capaces de reconocer las variadas tipologías de violencia evidentes en los textos, sin necesidad de que se muestre una batalla o agresión física. Asimismo, en cuanto a las imposiciones, de las conversaciones parece derivarse que al alumnado le resulta más fácil detectar su existencia cuando se conectan claramente con actos violentos y con rechazo a la divergencia o diversidad. Así, el grupo de tercero tuvo más dificultades para percibirlas en su álbum, Los Conquistadores (McKee, 2004), que el de quinto con el suyo, Felicio Rey del rebaño (Tallec, 2015). Estos obstáculos se evidencian en cómo interpreta el citado grupo de tercero el intercambio cultural entre el ejército conquistador y la población pretendidamente conquistada del pequeño país. Piensan que al General le parecerá bien y que su enfado se debe a no poder divertirse como sus soldados. Aunque se intenta que profundicen en la cuestión, solo un alumno identifica la causa, pero no consigue que su argumentación tenga la suficiente repercusión o continuidad en el resto:

Coordinadora: Y han venido nuevos. Pero se ha enfadado el General, no sé, no sé

Alumno 15: Se ha enfadado porque él como quería invadir ese pueblo, ellos no le han hecho caso al jefe, entonces ha traío otros

Alumna 3: El jefe se ha enfadado porque se portaban mal, y ha traído a otros

Alumno 22: Que el General les está diciendo a un grupo que se vaya para allá y a otro que se vaya para allá

Alumna 1: Deja allí a los soldados para saber lo que están haciendo y vigilarles, y ellos se van porque ya están cansados de estar allí

En ocasiones, se advierte que el pensamiento crítico se combina con el creativo, proponiendo soluciones alternativas para resolver el conflicto. Es el caso de segundo curso, cuando sugieren formas de que el protagonista pueda entrar en la casa con el resto de personajes:

Alumna 6: Que la única manera, o es cortárselos como ha dicho alumno 12, o que se arrugue y que se meta

$[\ldots]$

Alumno 3: Que también se puede enrollar y pasar

$[\ldots]$ 
Alumna 1: Que a lo mejor sus amigos círculos pueden intentar que lo pase, porque se ponen aquí y aprietan

Finalmente, el desacuerdo es un motor para la argumentación y el reajuste. La expresión de una opinión divergente obliga a dar razones para convencer al resto. El intercambio implica la introducción de variaciones en la idea inicial o la generación de nuevos argumentos para persistir en ella, como ocurrió con el grupo de sexto. El grupo de segundo también fue escenario de divergencias, con un alumno que se negaba a aceptar el pacto narrativo, lo que obligó al resto a pensar críticamente sobre el hecho literario:

\author{
Alumno 11: Que no es una casa, que es un papel \\ Alumno 7: Es un papel, pero lo usan como casa \\ Alumna: 2: Es un recortado \\ $[\cdots]$
}

\begin{abstract}
Alumno 11: Que no es una casa, que es un folio
Maestra: Es una casa, alumno 11, es un cuento
\end{abstract}

Alumno 21: ¿No ves que son como unos círculos, y un cuadrado recortado?

Alumna 1: Es de fantasía

\title{
4. Discusión y conclusiones
}

Como ya había sido puesto de relieve en las investigaciones que conforman el marco teórico, el enfoque dialógico ofrece indudables oportunidades de desarrollo del pensamiento crítico en el alumnado. Conversar sobre los álbumes permitió en este estudio respuestas más extensas y argumentadas por parte de los niños y niñas participantes. La creación de un clima de confianza, en el que todas las aportaciones se tenían por valiosas, provocó una expresión libre y segura de las opiniones. Se observó que no había temor en aportar una idea que contradijera las de otros, siempre de forma razonada, así como tampoco lo había en rectificar cuando el contraste evidenciaba la necesidad de hacerlo. Estos son aspectos clave para el correcto despliegue de la discusión en torno a los álbumes, y, claro está, para el progreso en el pensamiento crítico.

El hecho de compartir las ideas hace que unos puedan apoyarse en las de otros, ampliándolas, rebatiéndolas, descubriendo detalles que habían sido obviados, llegando a un pensamiento común más complejo que aquel que se produce de manera individual (Rincón, 2012). No obstante, la falta 
de hábito con respecto a este tipo de actividad, hace que las conversaciones sean en algunos momentos más de discusión y acumulativas, que exploratorias (Mercer, 1997). Se ve especialmente en los primeros cursos, donde el egocentrismo infantil todavía está muy presente. El hábito se crearía con una mayor frecuencia en las discusiones, y este sería uno de los déficits de este trabajo. Otro es la aplicación independiente en cada curso. Lo ideal sería una investigación longitudinal que pudiese mostrar el efecto acumulativo, como se ha visto en otros estudios (Evans, 2015).

De los resultados se extrae también la conclusión de que el pensamiento crítico es multiforme, yendo desde la puesta en jaque de la uniformidad a la toma de conciencia sobre la manipulación, pasando por el posicionamiento contra la atribución ilegítima del poder. Obviamente, la selección de los álbumes incide en la variedad de respuestas posibles. Y para seleccionar bien hay que conocer bien. Este conocimiento profundo es clave para tener confianza en una correcta guía de la discusión (Chambers, 2017; Roche, 2015).

Siguiendo con esta cuestión, las dificultades van a surgir de la necesidad de variar la perspectiva en la concepción de las dinámicas de aula. Implantar la discusión literaria en el proceso de enseñanza/aprendizaje requiere de reflexión sobre la práctica docente y disposición al cambio. Tres pueden ser los obstáculos que se presenten en las primeras aplicaciones: no crear oportunidades reales para que el alumnado converse sobre los álbumes; forzar las intervenciones y emitir juicios de valor; y falta de seguridad, que se traduce en un temor a no saber realizar las preguntas adecuadas, a no saber guiar al grupo, etc.

El enfoque dialógico se plantea, tras los resultados obtenidos, como una estrategia adecuada para avanzar tanto en la dimensión social del pensamiento crítico, gracias a la discusión en gran grupo, como en la individual, por la tarea posterior de escritura. Tarea que para Klooster (2001) sería la mejor para promover el citado pensamiento.

Pese a sus innegables limitaciones, esta investigación contribuye a evidenciar el potencial de la lectura dialógica para promover el sentido crítico en Educación Primaria. Dando cuenta de que en esta etapa también es posible hacerlo, y de que los álbumes son un recurso idóneo para ello.

\section{Referencias}

Alba Rico, S., Appadurai, A., Bauman, Z., Della Porta, D., Fraser, N., Garcés, M., Illouz, E., Krastev, I., Latour, B., Mason, P., Mishra, P., Misik, R., Nachtwey, O., Rendueles, C., Streeck, W., Van Reybrouck, D. y Žižek, S. (2017). El gran retroceso. Un debate internacional sobre el reto urgente de reconducir el rumbo de la democracia. Seix Barral. 
Albertos, D., y De la Herrán, A. (2018). Desarrollo del pensamiento crítico en estudiantes de educación secundaria: Diseño, aplicación y evaluación de un programa educativo. Profesorado: Revista de curriculum y formación del profesorado, 22(4), 269-285. https://doi.org/10.30827/profesorado.v22i4.8416

Alemagna, B. (2011). El país de las pulgas (B. Alemagna, Ilust.). Phaidon.

Álvarez-Álvarez, C., y Pascual-Díez, J. (2013). Estudio de caso sobre la formación de lectores críticos mediante textos literarios en la Educación Primaria. Ocnos: Revista De Estudios Sobre Lectura, (10), 27-53. https://doi.org/10.18239/ocnos_2013.10.02

Arizpe, E., y Styles, M. (2004). Lectura de Imágenes. Los niños interpretan textos visuales. Fondo de Cultura Económica.

Cali, D. (2008). El enemigo (S. Bloch, Ilust.). SM.

Cassany, D. (2004). Explorando las necesidades actuales de comprensión. Aproximaciones a la comprensión crítica. Lectura y Vida, 25(2), 6-23.

Cassany, D. (2013). Tras las líneas. Sobre la lectura contemporánea. Anagrama.

Cassany, D. (2017). Aproximaciones a la lectura crítica: teoría, ejemplos y reflexiones. Tarbiya. Revista de investigación e innovación educativa, (32), 113-132. https://revistas.uam.es/tarbiya/article/view/7275

Colomer, T. (2012). La literatura que acoge: un proyecto de investigación en las aulas. En T. Colomer y M. Fittipaldi (Coords.), La literatura que acoge. Inmigración y lectura de álbumes (pp. 7-25). Banco del Libro y GRETEL.

Courtland, M. C., y González, I. (2013). Imagining the Possibilities: The Pedagogical Potential of Diverse Canadian Picture Books. En J. Johnston and J. Bainbridge (Eds.) Reading Diversity through Canadian Picture Books. Preservice Teachers Explore Issues of Identity, Ideology, and Pedagogy. University of Toronto Press.

Cunningham, K., y Enriquez, G. (2013). Bridging Core Readiness with Social Justice Through Social Justice Picture Books. New England Reading Association Journal, 48(2), 28-87.

Da Mota, H. (2020). Citizenship and Language Education. Picture Books: New Opportunities for Young Teenagers. (Tesis doctoral, Universidade Nova de Lisboa). http://hdl.handle.net/10362/100500

Ellis, G., y Mourão, S. (2021, 7 de junio). Demystifying the read-aloud in early English language learning. [Vídeo]. YouTube. https://www.youtube.com/watch?v=3LTyWxCikMI\&t=916s

European Commission/EACEA/Eurydice (2017). Citizenship Education at School in Europe - 2017. Eurydice Report. Publications Office of the European Union. https://doi.org/10.2797/612559

Evans, J. (Ed.). (2015). Challenging and Controversial Picturebooks. Creative and critical responses to visual texts. Routledge.

Freebody, P., y Luke, A. (1991). Literacies programs: Debates and demands in cultural contexts. Prospects, 5(3), 7-16.

Giroux, H. (1990). Los profesores como intelectuales: hacia una pedagogía crítica del aprendizaje. Paidós.

Guzmán-Cedillo, Y. I. y Flores, R. C. (2020). La competencia argumentativa como meta en contextos educativos. Revisión de la literatura. Educar, 56(1) 15-34. https://doi.org/10.5565/rev/educar.1009

Jeffers, O. (2015). Los Huguis en El jersey nuevo (O. Jeffers, Ilust.). Andana.

Jurado, F. (2008). La formación de lectores críticos desde el aula. Revista Iberoamericana de Educación, 46, 89105.

Kalogirou, T., y Malafantis, K. (2012). Do I dare/disturb the universe? Critical Pedagogy and the ethics of resistance to and engagement with literature. Journal for Critical Education Policy Studies, 10(1), $265-284$. 
Kalogirou, T., y Malafantis, K. (2014). Negotiating Miranda's vision in the classroom: Critical encounters with literature, from archetypal symbolism to dystopian fantasy. En A. Reyes-Torres, L. S. Villacañas-de-Castro y B. Soler-Pardo (Eds.), Thinking through Children's Literature in the Classroom (pp. 129-149). Cambridge Scholar Publishing.

Klooster, D. (2001). What is Critical Thinking? Thinking Classroom. A Journal of Reading, Writing and Critical Reflection, 2(2), 36-40.

Martínez, P., Ballester, J., e Ibarra, N. (2018). Estudio cuasiexperimental para el análisis del pensamiento crítico en secundaria. Revista Electrónica de Investigación Educativa, 20(4), 123-132. https://doi.org/10.24320/redie.2018.20.4.1705

McDaniel, C. (2004). Critical literacy: A questioning stance and the possibility for change. The Reading Teacher, $57(5), 472-481$.

McDaniel, C. (2006). Critical literacy: A way of thinking, a way of life. Peter Lang.

McKee, D. (2004). Los conquistadores (D. McKee, llust.). Kókinos.

Mercer, N. (1997). La construcción guiada del conocimiento. El habla de profesores y alumnos. Paidós.

Morales, J. (2018). Aportes de Paulo Freire a la Investigación y a la Lectura Crítica. Revista Internacional de Educación para la Justicia Social, 7(2), 175-192. https://doi.org/10.15366/riejs2018.7.2.010

Mounk, Y. (2018). El pueblo contra la democracia. Por qué nuestra libertad está en peligro y cómo salvarla. Paidós.

Munita, F., y Manresa, M. (2012). La mediación en la discusión literaria. En T. Colomer y M. Fittipaldi (Coords.), La literatura que acoge. Inmigración y lectura de álbumes (pp. 119-143). Banco del libro y GRETEL.

Peterson, R., y Eeds, M. (2007). Grand Conversations. Literature Groups in Action. Scholastic.

Phipps, H. (2016). Children speaking with children Visualizing Engagement Through Contemporary Canadian Picture Books in French Classrooms. (Tesis doctoral, McGill University). http://digitool.library.mcgill.ca/R/?func=dbin-jump-full\&object_id=145374

Real Decreto 126/2014, de 28 de febrero, por el que se establece el currículo básico de la Educación Primaria. Boletín Oficial del Estado, 52, 1 de marzo de 2014, pp. 19349-19420.

Rincón, A. (2012). De la imagen a la palabra. Conversaciones literarias en el aula de acogida. En T. Colomer y M. Fittipaldi (Coords.), La literatura que acoge. Inmigración y lectura de álbumes (pp. 197-205). Banco del libro y GRETEL.

Ruillier, J. (2005). Por cuatro esquinitas de nada (J. Ruillier, Ilust.). Juventud.

Saldaña, A. (2020). Sobre literatura y pensamiento crítico en la posmodernidad. Tropelías. Revista de Teoría de la Literatura y Literatura Comparada, número extraordinario 6, 86-103. https://doi.org/10.26754/ojs_tropelias/tropelias.202064232

Santisteban, A. (2004). Formación de la ciudadanía y educación política. En M.I., Vera y D., Pérez (coord.), Formación de la ciudadanía: las TICS y los nuevos problemas. Universidad de Alicante/Asociación Universitaria de Profesores de Didáctica de las Ciencias Sociales.

Stufflebeam, D., y Shinkfield, A. (1995). Evaluación sistemática. Guía teórica y práctica. Paidós y Ministerio de Educación y Ciencia.

Tallec, O. (2015). Felicio Rey del rebaño (O. Tallec, Ilust.). Algar Editorial.

Todorov, T. (2016). Los enemigos íntimos de la democracia. Galaxia Gutenberg.

Valadier, P. (2017). La posverdad, peligro para la democracia. Revista De Fomento Social, (286), $297-304$. https://doi.org/10.32418/rfs.2017.286.1426 


\section{How to cite this paper:}

Ruiz-Guerrero, L.; Hoster-Cabo, B. \& Molina-Puche, S. (2021). Lectura dialógica de álbumes ilustrados para fomentar el pensamiento crítico: un enfoque para Educación Primaria. Journal of Literary Education, (5), 33-53.

https://doi.org/10.7203/JLE.5.21057 